\title{
The utility of early post-liver transplantation model for end-stage liver disease score in prediction of long-term mortality
}

\author{
Habibollah Dashtia,b, Amirpasha Ebrahimia, Niloofar Razavi Khorasanii ${ }^{b}$, Bobak Moazzami ${ }^{b}$, \\ Fatemeh Khojastehc, Sediqe Hoseinic, Ali Jafarian ${ }^{a, b}$
}

Imam Khomeini Hospital Complex, Tehran University of Medical Sciences, Tehran, I.R. Iran

\section{Abstract}

\begin{abstract}
Background Little is known about the prognostic ability of post-liver transplantation (LT) model for end-stage liver disease (MELD) score measurement in assessing long-term outcomes. The aim of the present study was to investigate this possible relationship.
\end{abstract}

\begin{abstract}
Methods In this retrospective cohort study, the medical records of LT recipients operated under a LT program were reviewed. The accuracy of post-operation MELD score for predicting mortality was evaluated based on receiver operating characteristic (ROC) curves. Univariate and Cox proportional hazard regression models were used to assess the risk factors associated with mortality.

Results Eight hundred twenty-six consecutive LT recipients were included in the study. The areas under the ROC curve on postoperative days (POD) 5 and 9 for predicting 1-year mortality were 0.712 (95\% confidence interval [CI] 0.614-0.811) and 0.682 (95\%CI 0.571-0.798), respectively. A cutoff point of 14.5 was obtained for MELD score on POD5 that significantly differentiated between survivors and non-survivors with a sensitivity of $69.8 \%$ (95\%CI 50.7-83.1) and a specificity of $57.2 \%$ (95\%CI 50.6-63.6). In the Cox multivariate analysis, factors including MELD score on POD5 (hazard ratio [HR] 1.83, 95\%CI 1.07-3.12; $\mathrm{P}=0.026$ ), pre-transplant MELD (HR 1.064, 95\%CI 1.025-1.104; $\mathrm{P}=0.001)$ and operation duration ( $\min )$ (HR 1.004, 95\%CI 1.003-1.006; $\mathrm{P}=0.013$ ) were identified as independent risk factors for predicting overall survival.
\end{abstract}

Conclusion The immediate postoperative MELD scores after LT may be of value in predicting mortality and could be used as a tool for postoperative risk assessment of patients.

Keywords Liver transplantation, cirrhosis, model for end-stage liver disease score, outcome

Ann Gastroenterol 2019; 32 (6): 1-9

\section{Introduction}

Liver transplantation (LT) has been considered as a curative treatment modality for patients with end-stage

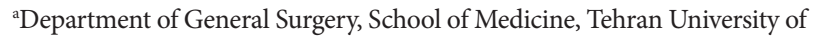
Medical Sciences (Halibollah Dashti, Amirpasha Ebrahimi, Ali Jafarian);

bLiver Transplantation Research Center, Imam Khomeini Hospital Complex, Tehran University of Medical Sciences (Habibollah Dashti, Niloofar Razavi Khorasani, Bobak Moazzami, Ali Jafarian); 'Tehran University of Medical Sciences (Fatemeh Khojasteh, Sediqe Hoseini), Iran

Conflict of Interest: None

Correspondence to: Ali Jafarian, MD, Professor and Chief, Division of HPB and Liver Transplantation, Imam Khomeini Hospital Complex, Keshavarz Blvd, Tehran, Iran, e-mail: jafarian@tums.ac.ir

This research has been supported by Tehran University of Medical Sciences \& Health Services

Received 14 June 2019; accepted 28 August 2019; published online 1 October 2019

DOI: https://doi.org/10.20524/aog.2019.0420 liver disease [1]. Despite recent advances in both surgical techniques and the medical management of patients, short- and long-term adverse outcomes following LT still represent the major source of morbidity and mortality [2]. The occurrence of postoperative complications depends on several donor- and recipient-related risk factors and may have a substantial impact on outcomes and costs [3-5]. Thus, postoperative patient survival is affected by preoperative factors related to both donor and recipient, including recipient age (older than 60 years), comorbid conditions (e.g., diabetes, renal failure, cardiac or pulmonary disease, malnutrition) and the quality of the donor liver graft, as well as the complexity of the surgical procedure [6-9].

The model for end-stage liver disease (MELD) score was originally designed and validated to predict mortality in patients with portal hypertension who developed complications due to transjugular portosystemic shunt [10]. Subsequently, this scoring system was modified and used as the standard tool in prioritizing candidates for LT [11,12]. In addition, previous studies have indicated that the MELD score is a reliable prognostic model for 3-month mortality among patients 
awaiting LT $[11,13]$. Although the utility of the MELD score in predicting pre-transplant mortality has been extensively investigated, there are only limited data concerning its relation with post-transplantation outcomes. Recently, the MELD score on postoperative day (POD) 5 was found to be significantly associated with the risk of death after hepatectomy [14]. This finding may lead to a better assessment of the severity of clinical risk scores and could be used to guide clinical decision-making concerning the medical management of patients. Therefore, in the current study we aimed to evaluate the prognostic ability of the post-transplantation MELD score in the prediction of longterm outcomes following LT.

\section{Patients and methods}

\section{Patients}

In this single-center retrospective cohort study, the medical records of 904 consecutive adult patients who underwent LT under a LT program (affiliated to Tehran University of Medical Sciences) between April 2001 and December 2018 were evaluated. Data were extracted from the LT research center database. All patients included were over 18 years of age. Patients listed for re-transplantation were excluded, as were those with acute liver failure. Demographic data were collected, including recipient age at the time of LT, donor age and sex, etiology of liver disease and indication for LT. Operation variables, including warm and cold ischemic time, duration of surgery, and quantities of packed red blood cells, fresh frozen plasma (FFP) and platelets transfused during surgery were gathered. The severity of liver cirrhosis was assessed using the Child-Pugh and MELD scores.

This study was approved by the local ethics committee of the Tehran University of Medical Science. The study was conducted in accordance with the Declaration of Helsinki and other applicable guidelines, laws and regulations [15].

\section{Measurements}

Clinical information and laboratory values, including routine biochemical analysis (complete blood count), liver enzyme and function tests, serum total bilirubin level, serum creatinine levels and international normalized ratio (INR), were measured and recorded for all patients. MELD score was calculated using the following formula [16]:

$3.78 \times \ln ($ T-bilirubin $[\mathrm{mg} / \mathrm{dL}])+9.57 \times \ln ($ creatinine $[\mathrm{mg} / \mathrm{dL}])+11.20 \times \ln (\mathrm{INR})+6.43 \times(0$ if cholestatic or alcoholic, 1 otherwise).

The MELD score was measured daily from the first day after surgery until 20 days after transplantation. The MELD scores on POD 5, 9 and 15 were the highest peaks during the followup period. Therefore, the effect of applying post-operation MELD scores on POD 5, 9 and 15 as a predictor of mortality was examined using receiver operating characteristic (ROC) curves.

\section{Surgical procedure and immunosuppression protocol}

In this cohort, all patients who were included had undergone LT by the same surgical transplant team and were followed under the same surgical protocol. Liver were harvested from brain-dead donors and preserved in University of Wisconsin solution prior to transplantation. Our routine procedure is a piggyback technique with cavo-caval anastomosis in side-toside and end-to-end fashion. In patients with an enlarged liver associated with Budd-Chiari syndrome and a diagnosis of primary sclerosing cholangitis, the standard surgical technique was performed with cavo-caval end-to-end anastomosis. The end-to-end (89.2\%) and Roux-en-Y (10.8\%) techniques were used for the reconstruction of bile ducts.

The immunosuppression protocol was initiated with one gram methylprednisolone administration concurrently with the placement of the liver in the patient's body. Postoperatively, patients received conventional daily divided immunosuppressive doses of $200 \mathrm{mg}$ methylprednisolone, tapered gradually during the first week, and then continued with $20 \mathrm{mg}$ oral prednisolone. Oral mycophenolate mofetil and tacrolimus doses were administered on the first and second postoperative days, respectively, with a gradual increase in dosage until reaching an optimal dose. Patients were followed for the first month following surgery and closely monitored for any signs of infectious and noninfectious complications.

\section{Statistical analysis}

Continuous variables were examined for a normal distribution using the Shapiro-Wilk test. The $t$-test was used for independent numeric variables, while for independent nominal variables Fisher's exact test or the Mann-Whitney $U$ test were used as indicated. Continuous variables were reported as mean \pm standard deviation (SD) if normally distributed, or the median and $25^{\text {th }}$ and $75^{\text {th }}$ percentiles if non-normally distributed. In order to identify preoperative and postoperative risk factors for long-term mortality, univariate and multivariate logistic regression analyses were carried out using the following parameters: age, sex, etiology of cirrhosis, pre-operation MELD score, pre-operation Child-Pugh score, and the postoperative MELD scores. The impact of perioperative and postoperative variables on overall survival was examined using a univariate and multivariate Cox proportional hazards regression model. Survival analysis carried out using the Kaplan-Meier method. The log-rank test was used to compare the survival times between groups. The accuracy of pre-transplant and post-LT MELD scores for predicting mortality was evaluated based on ROC curves. The area under the ROC curve (AUROC) was calculated with $95 \%$ confidence intervals (CI). Values below 0.7 were suggestive of poor predictive value, while scores above 0.70 were considered a reliable test, while an AUROC of greater than 0.80 indicated excellent predictive accuracy [17]. The cutoff values were defined in line with the optimal sensitivity and specificity. All statistical analyses were performed using SPSS 18.0 (SPSS Inc.; Chicago, IL). Statistical significance was defined as $\mathrm{P}<0.05$. 


\section{Results}

\section{Characteristics of patients}

A total of 826 patients were eligible to be included in the final analysis. During the follow-up period, 685 patients $(80.7 \%)$ were still alive. Mean age at the time of operation was $43.44 \pm 13.98$ years for all transplant candidates. The median follow-up time was 1175 days (range 5-4111 days). The most common etiology of liver cirrhosis was cryptogenic (18\%), followed by hepatitis B (14\%), autoimmune (13.9\%), hepatitis C (13.4\%), and primary sclerosing cholangitis (9.9\%). The preoperative MELD scores were significantly higher among non-surviving patients compared to survivors (23.01 \pm 7.04 vs. $20.28 \pm 5.78 ; \mathrm{P}=0.003$ ). Similarly, non-surviving patients had higher MELD scores on POD5 and POD9 than survivors, whereas the postoperative MELD score on POD15 was not significantly different between non-survivors and survivors $(16.89 \pm 8.32$ vs. $12.98 \pm 5.55 ; \mathrm{P}<0.001$ for POD5, $14.57 \pm 9.95$ vs. $10.75 \pm 5.55$; $\mathrm{P}<0.001$ for $\mathrm{POD} 9$ and
$12.18 \pm 5.71$ vs. $12.33 \pm 5.82 ; \mathrm{P}=0.92$ for $\mathrm{POD} 15)$. Both cold ischemic time and surgery duration were significantly longer among patients who died $(360.19 \pm 158.32$ vs. $305.49 \pm 86.89$, $\mathrm{P}<0.001$ and $394.37 \pm 127.32$ vs. $324.91 \pm 83.28 ; \quad \mathrm{P}<0.001$, respectively). Details of the postoperative laboratory evaluations are given in Table 1.

\section{Sensitivity and specificity of post-operation MELD scores by AUROC}

AUROC analysis was applied to determine the sensitivities and specificities of optimal cutoff points for the post-operation MELD score for predicting patients' survival. The AUROC curve obtained by plotting different cutoffs is shown in Fig. 1. The AUROC values for POD5 and POD9 during 5 years of follow up were 0.638 (95\%CI $0.557-0.721)$ and 0.621 (95\%CI 0.522-0.717), respectively. A cutoff point of 14 was obtained for MELD score on POD5. A cutoff point of 11.5 for the MELD

Table 1 Differences in clinical characteristics and laboratory findings between survivors and non-survivors of liver transplantation

\begin{tabular}{|c|c|c|c|c|}
\hline Parameters & Total $(n=826)$ & Non-survivors $(n=141)$ & Survivors $(\mathrm{n}=685)$ & P-value \\
\hline Donor age (years) & $35.11 \pm 13.42$ & $37.59 \pm 13.84$ & $34.55 \pm 13.25$ & 0.025 \\
\hline Recipient age (years) & $43.44 \pm 13.98$ & $43.88 \pm 14.07$ & $43.17 \pm 13.97$ & 0.59 \\
\hline Recipient sex (m/f) & $483 / 343$ & $86 / 55$ & $397 / 288$ & 0.72 \\
\hline Cold ischemic time (min) & $297.87 \pm 80.48$ & $311.21 \pm 102.94$ & $294.99 \pm 74.53$ & 0.035 \\
\hline Warm ischemic time (min) & $25.58 \pm 15.53$ & $27.02 \pm 18.78$ & $25.32 \pm 14.81$ & 0.274 \\
\hline Surgery duration (min) & $299.79 \pm 83.82$ & $325.94 \pm 113.91$ & $294.11 \pm 74.65$ & $<0.001$ \\
\hline Number of PRC used & $4.22 \pm 3.18$ & $5.47 \pm 5.57$ & $3.72 \pm 2.71$ & $<0.001$ \\
\hline Number of FFP used & $3.73 \pm 0.94$ & $5.41 \pm 1.99$ & $3.23 \pm 0.62$ & $<0.001$ \\
\hline Number of PLT used & $3.76 \pm 0.86$ & $4.71 \pm 1.82$ & $3.43 \pm 0.76$ & $<0.001$ \\
\hline Hospital stay (days) & $15.65 \pm 10.88$ & $18.56 \pm 16.95$ & $15.07 \pm 9.18$ & 0.071 \\
\hline $\begin{array}{l}\text { Pre-transplant MELD } \\
\text { score }\end{array}$ & $21.74 \pm 6.13$ & $23.01 \pm 7.04$ & $20.28 \pm 5.78$ & 0.003 \\
\hline $\begin{array}{l}\text { Pre-transplant Child-Pugh } \\
\text { score }\end{array}$ & $9.91 \pm 2.04$ & $10.07 \pm 2.11$ & $9.87 \pm 2.03$ & 0.41 \\
\hline $\begin{array}{l}\text { POD } 5 \\
\text { Platelets }\left(\times 10^{3} / \mu \mathrm{L}\right)(\mathrm{IQR}) \\
\text { WBC }\left(/ \mathrm{mm}^{3}\right)(\mathrm{IQR}) \\
\text { Hemoglobin }(\mathrm{g} / \mathrm{dL}) \\
\text { MELD }\end{array}$ & $\begin{array}{c}45.0(30-74) \\
5400(3400-8200) \\
9.29 \pm 1.54 \\
13.73 \pm 6.35\end{array}$ & $\begin{array}{c}36(25-58) \\
5850(3700-9875) \\
9.81 \pm 1.92 \\
16.89 \pm 8.32\end{array}$ & $\begin{array}{c}47(31-81) \\
5300(3300-7900) \\
9.32 \pm 1.44 \\
12.98 \pm 5.55\end{array}$ & $\begin{array}{c}\mathbf{0 . 0 2 1}^{\star} \\
\mathbf{0 . 0 5}^{\star} \\
0.534 \\
<\mathbf{0 . 0 0 1}\end{array}$ \\
\hline $\begin{array}{l}\text { POD } 9 \\
\text { Platelets }\left(\times 10^{3} / \mu \mathrm{L}\right)(\mathrm{IQR}) \\
\text { WBC }\left(/ \mathrm{mm}^{3}\right)(\mathrm{IQR}) \\
\text { Hemoglobin }(\mathrm{g} / \mathrm{dL}) \\
\text { MELD }\end{array}$ & $\begin{array}{c}107(69.5-158.0) \\
8800(6200-12300) \\
10.38 \pm 1.69 \\
12.29 \pm 5.74\end{array}$ & $\begin{array}{c}81(39-113) \\
8300(5200-13050) \\
10.08 \pm 1.78 \\
14.57 \pm 9.95\end{array}$ & $\begin{array}{c}114(72-169) \\
8800(6325-12175) \\
10.45 \pm 1.67 \\
10.75 \pm 5.55\end{array}$ & $\begin{array}{c}\mathbf{0 . 0 0 1}^{\star} \\
0.659^{\star} \\
0.202 \\
<\mathbf{0 . 0 0 1}\end{array}$ \\
\hline $\begin{array}{l}\text { POD } 15 \\
\text { Platelets }\left(\times 10^{3} / \mu \mathrm{L}\right)(\mathrm{IQR}) \\
\text { WBC }\left(/ \mathrm{mm}^{3}\right)(\mathrm{IQR}) \\
\text { Hemoglobin }(\mathrm{g} / \mathrm{dL}) \\
\text { MELD }\end{array}$ & $\begin{array}{c}147(91-213) \\
8100(5900-11300) \\
9.69 \pm 1.46 \\
12.29 \pm 5.74\end{array}$ & $\begin{array}{c}117(66-197) \\
7700(5700-9650) \\
9.16 \pm 1.01 \\
12.18 \pm 5.71\end{array}$ & $\begin{array}{c}156(94-233) \\
8200(6075-11550) \\
9.84 \pm 1.54 \\
12.33 \pm 5.82\end{array}$ & $\begin{array}{c}0.212^{*} \\
0.779^{*} \\
\mathbf{0 . 0 4 9} \\
0.92\end{array}$ \\
\hline
\end{tabular}

${ }^{\mathrm{a}} \mathrm{P}<0.05$ was considered significant

${ }^{*}$ The between-group comparison was made using Mann-Whitney $\mathrm{U}$ test

POD, postoperative day; WBC, white blood cells; PRC, packed red cells; FFP, fresh frozen plasma; PLT, platelet; IQR, interquartile range; MELD, model for end-stage liver disease 
A

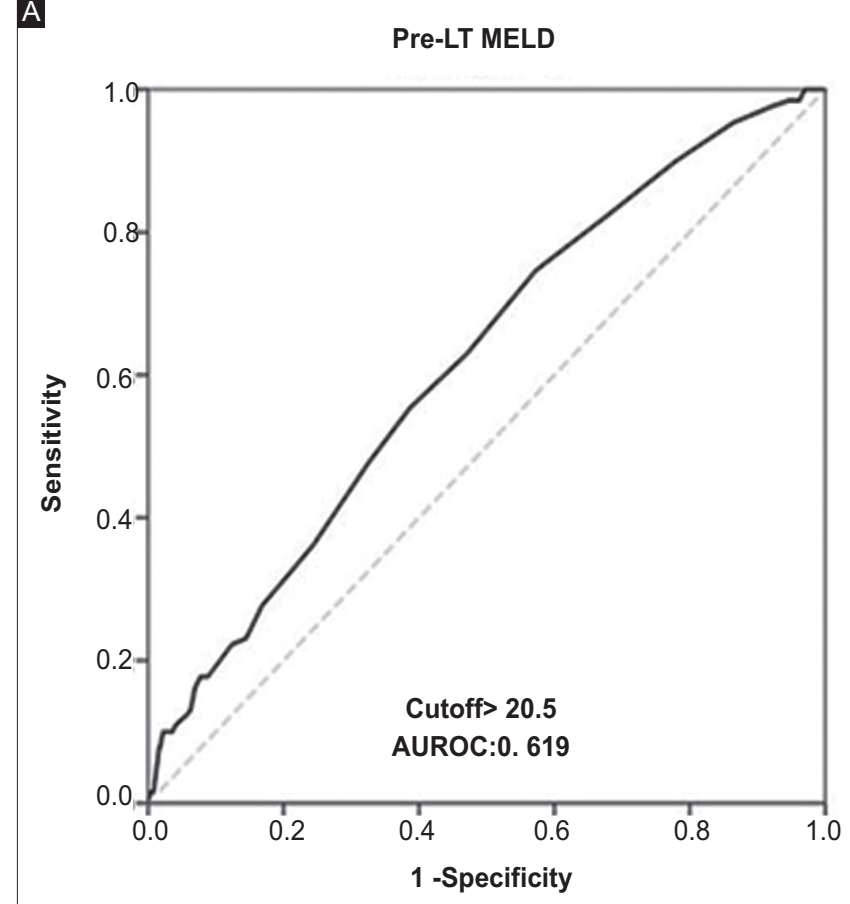

C

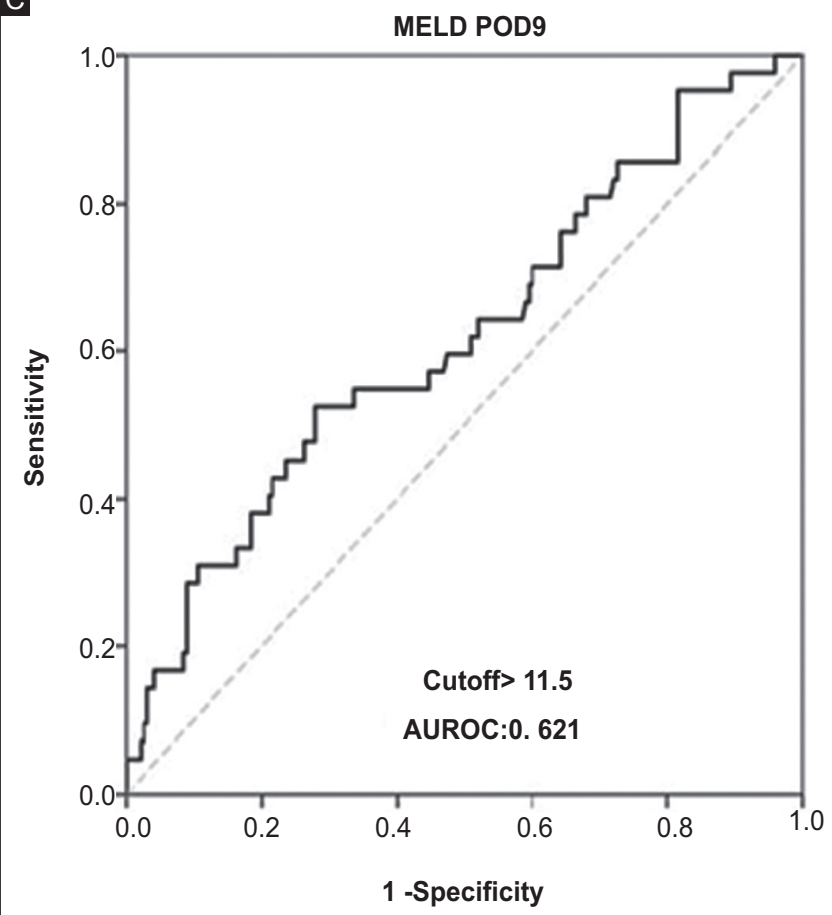

B

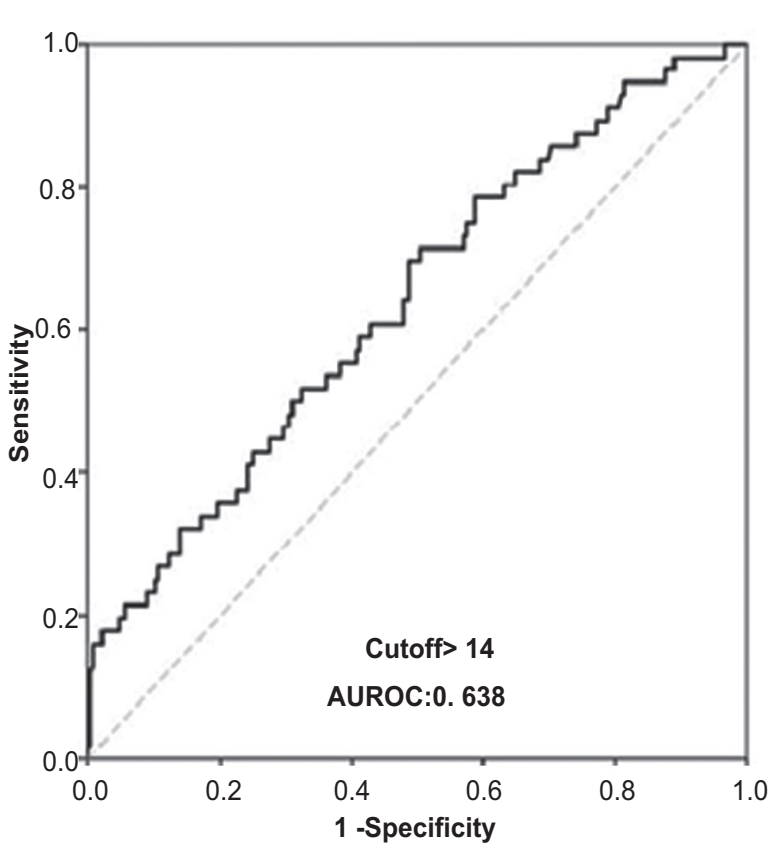

D

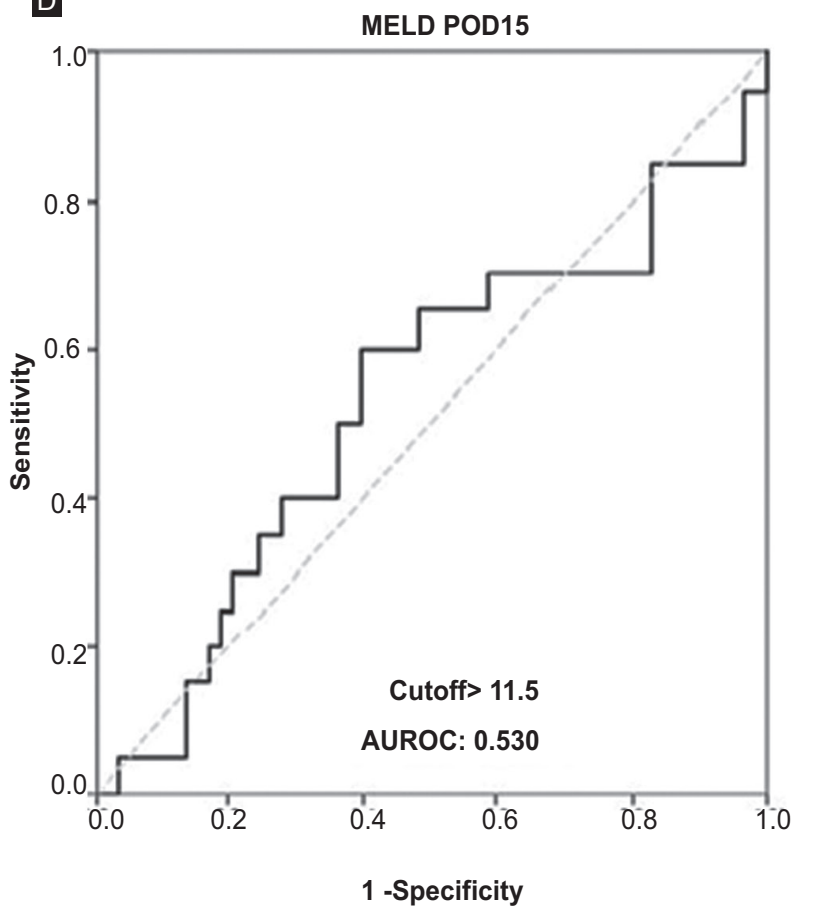

Figure 1 The AUROC for MELD score prior to transplantation (A) and on POD5 (B), POD9 (C) and POD15 (D) in relation to survival over 5 years of follow up AUROC, area under the receiver operating characteristic curve; MELD, model for end-stage liver disease; POD postoperative day

score on POD9 was found to be optimal for predicting mortality. However, the sensitivity and specificity of these cutoff values reveal the limitations of this test in terms of differentiating between patients who died and those who survived (POD5 MELD score: sensitivity 68.4\%, specificity 63.0\%; POD9 MELD score: sensitivity $64.5 \%$, specificity $63.7 \%$ ).
When patients who were followed-up for more than 1 year were excluded, the AUROC values for POD5 and 9 were substantially increased to 0.712 (95\%CI 0.614-0.811) and 0.682 (95\%CI 0.571-0.798), respectively. A cutoff point of 14.5 was obtained for MELD score on POD5, which significantly differentiated between surviving and non-surviving patients 
during one year of follow up (Fig. 2). The diagnostic performance of pre- and post-LT MELD scores at different time points during follow up is shown in Table 2.

\section{Survival analysis}

The median time to death was 23 days, ranging from day 5 of transplantation to 3123 days following surgery. The overall mortality rate among patients was $13.6 \%$. The main cause of death was sepsis (41.8\%), followed by hepatic artery thrombosis $(6.3 \%)$ and pneumonia (6.3\%). Other causes of death are listed in Table 3. A significant survival difference between patients with POD5 MELD score 14 or below was observed, as illustrated by the Kaplan-Meier survival curve (log-rank test $\mathrm{P}<0.001)$. The 1-, 3-, 5- and 8-year survival rates were $82.8 \%, 80.6 \%, 77.2 \%$ and $76.3 \%$, respectively (Fig. 3).

\section{Multivariate analysis of prognostic factors for survival}

Univariate logistic regression analysis identified the following parameters to be significantly associated with mortality: donors' age, pre-transplant MELD score, POD5 MELD score, POD9 MELD score, quantities of packed red cells, platelets and FFP used, cold ischemic time duration, and operation duration (Table 4). Multivariate logistic regression analysis found only the POD5 MELD score $(\mathrm{P}=0.014)$ and operation duration $(\mathrm{P}=0.038)$ to be independent risk factors for mortality. In the Cox multivariate analysis (a stepwise forward conditional method), factors including MELD score on POD5 (hazard ratio [HR] 1.83, 95\%CI 1.07-3.12; $\mathrm{P}=0.026$ ), pre-LT MELD score (HR 1.064, 95\%CI 1.025-1.104; $\mathrm{P}=0.001$ ) and operation duration (min) (HR 1.004, 95\%CI 1.003-1.006; $\mathrm{P}=0.013$ ) were identified as independent risk factors for predicting overall survival (Table 5).

\section{Discussion}

Over the last three decades, survival following LT has significantly improved and has led to a steadily increasing demand for adult LT procedures. Therefore, there is an urgent need to construct a robust prognostic tool to optimize the distribution of scarce organs and offer them to appropriate candidates. MELD score has been used as a gold standard for predicting wait-list mortality in candidates with end-stage liver disease [18]. In addition, it was also shown that the posttransplant MELD score could be used as a prognostic tool in patients who underwent LT [19]. The aim of the present study was to measure MELD scores within the first 2 weeks following surgery and evaluate their ability to predict postoperative outcomes. Our results suggest that MELD on POD5 was independently associated with 1-year mortality; however, a cutoff point of 14.5 yielded an AUROC of only $~ 0.71$, indicating that MELD on POD5 was a poor predictor of post-transplant survival. To the best of our knowledge, the present study is the largest to examine the utility of immediate post-transplant MELD score in predicting the survival of LT patients.

Table 2 Diagnostic performance of MELD score for predicting mortality at 6 month, 12 month and 5 years of follow up

\begin{tabular}{lccccccc}
\hline Variables & $\begin{array}{c}\text { Optimal } \\
\text { cutoff }\end{array}$ & $\begin{array}{c}\text { Sensitivity } \\
(95 \% \mathrm{CI})\end{array}$ & $\begin{array}{c}\text { Specificity } \\
(95 \% \mathrm{CI})\end{array}$ & $\begin{array}{c}\text { PPV } \\
(95 \% \mathrm{CI})\end{array}$ & $\begin{array}{c}\text { NPV } \\
(95 \% \mathrm{CI})\end{array}$ & $\begin{array}{c}\text { LR }+ \\
(95 \% \mathrm{CI})\end{array}$ & $(95 \% \mathrm{CI})$ \\
\hline
\end{tabular}

Follow up at 6 months

\begin{tabular}{lccccccc}
\hline MELD pre LT & 21.5 & $47.6(36.6-58.8)$ & $67.5(63.7-71.1)$ & $16.2(11.8-21.4)$ & $90.7(87.7-93.2)$ & $1.46(1.14-1.88)$ & $0.78(0.63-0.96)$ \\
MELD POD5 & 15.5 & $60.0(40.6-77.3)$ & $70.6(66.4-76.4)$ & $20.7(12.7-30.7)$ & $93.3(88.5-96.5)$ & $2.04(1.44-2.91)$ & $0.57(0.36-0.88)$ \\
MELD POD9 & 10.5 & $70.8(48.9-87.4)$ & $51.1(43.7-58.4)$ & $15.5(9.3-23.6)$ & $93.3(86.6-97.3)$ & $1.45(1.08-1.94)$ & $0.57(0.30-1.08)$ \\
MELD POD15 & 12.5 & $41.7(35.8-60.3)$ & $63.8(50.1-76.0)$ & $19.2(6.6-39.4)$ & $84.1(69.9-93.4)$ & $1.15(0.54-2.44)$ & $0.91(0.55-1.53)$ \\
\hline
\end{tabular}

Follow up at 12 months

\begin{tabular}{lccccccc}
\hline MELD pre LT & 21.5 & $49.5(39.2-59.8)$ & $67.5(63.7-71.1)$ & $18.8(14.2-24.2)$ & $89.7(86.7-92.3)$ & $1.52(1.21-1.91)$ & $0.75(0.61-0.92)$ \\
MELD POD5 & 14.5 & $69.8(50.7-83.1)$ & $57.2(50.6-63.6)$ & $19.2(12.7-27.2)$ & $92.5(86.9-96.2)$ & $1.60(1.23-2.10)$ & $0.55(0.33-0.91)$ \\
MELD POD9 & 10.5 & $67.9(47.6-84.1)$ & $51.1(43.7-58.4)$ & $17.0(10.5-25.2)$ & $91.5(84.5-96.0)$ & $1.39(1.03-1.86)$ & $0.63(0.36-1.10)$ \\
MELD POD15 & 8.5 & $66.7(41.3-79.7)$ & $19.0(9.9-31.4)$ & $17.5(8.7-29.9)$ & $68.8(41.3-89.0)$ & $0.82(0.52-1.20)$ & $1.76(0.72-4.29)$ \\
\hline
\end{tabular}

Follow up at 5 years

\begin{tabular}{lccllllll}
\hline MELD pre LT & 20.5 & $57.6(47.2-67.50$ & $61.3(57.4-65.1)$ & $18.8(14.6-23.7)$ & $90.3(87.1-92.9)$ & $1.49(1.22-1.81)$ & $0.69(0.55-0.88)$ \\
MELD POD5 & 14 & $68.4(51.3-82.5)$ & $63.0(56.5-69.2)$ & $23.0(15.6-31.9)$ & $92.5(87.3-96.1)$ & $1.85(1.41-2.43)$ & $0.50(0.31-0.81)$ \\
MELD POD9 & 11.5 & $64.5(45.4-80.8)$ & $63.7(56.4-70.5)$ & $22.5(14.3-32.6)$ & $91.7(85.6-95.8)$ & $1.78(1.29-2.45)$ & $0.56(0.34-0.91)$ \\
MELD POD15 & 11.5 & $52.9(27.8-77.1)$ & $55.2(41.5-68.3)$ & $25.7(12.5-43.4)$ & $80.0(64.4-90.9)$ & $1.18(0.69-2.01)$ & $0.85(0.35-1.49)$ \\
\hline
\end{tabular}

Optimal cutoff points gave the highest total sensitivity and specificity

$P P V$, positive predictive value; NPV, negative predictive value; $L R$, likelihood ratio; MELD, model for end-stage liver disease; POD, post-operation day 
A

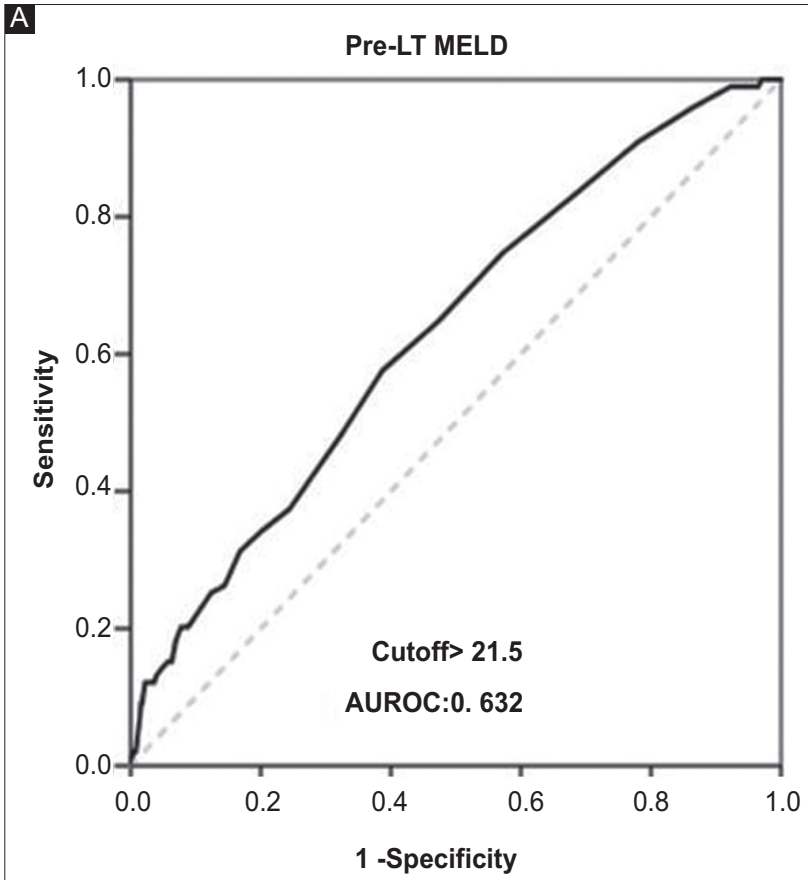

C

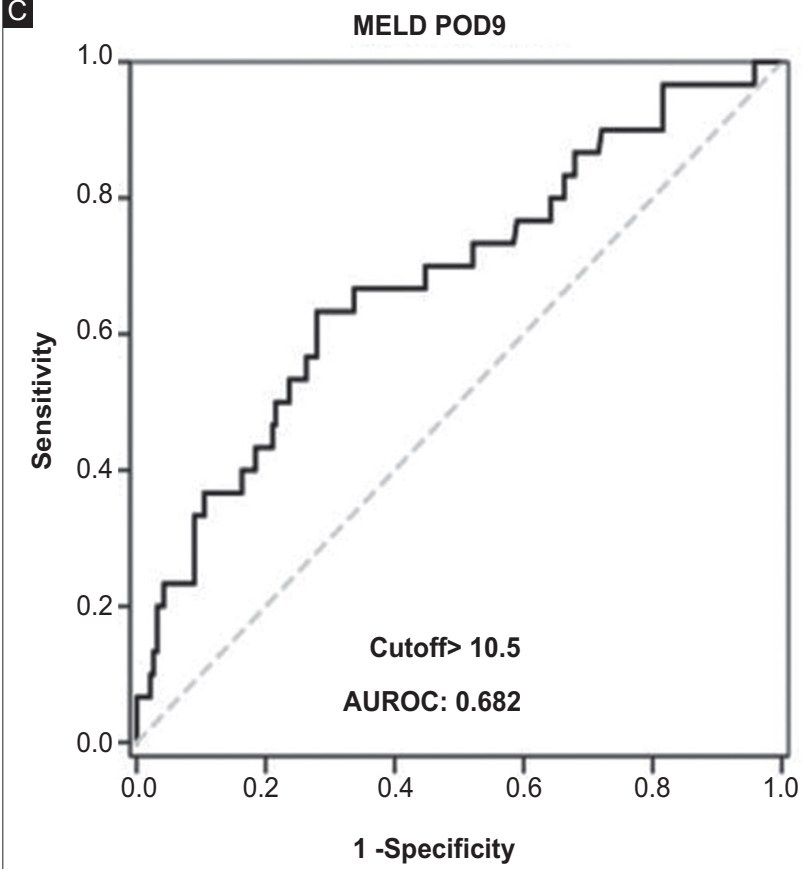

B
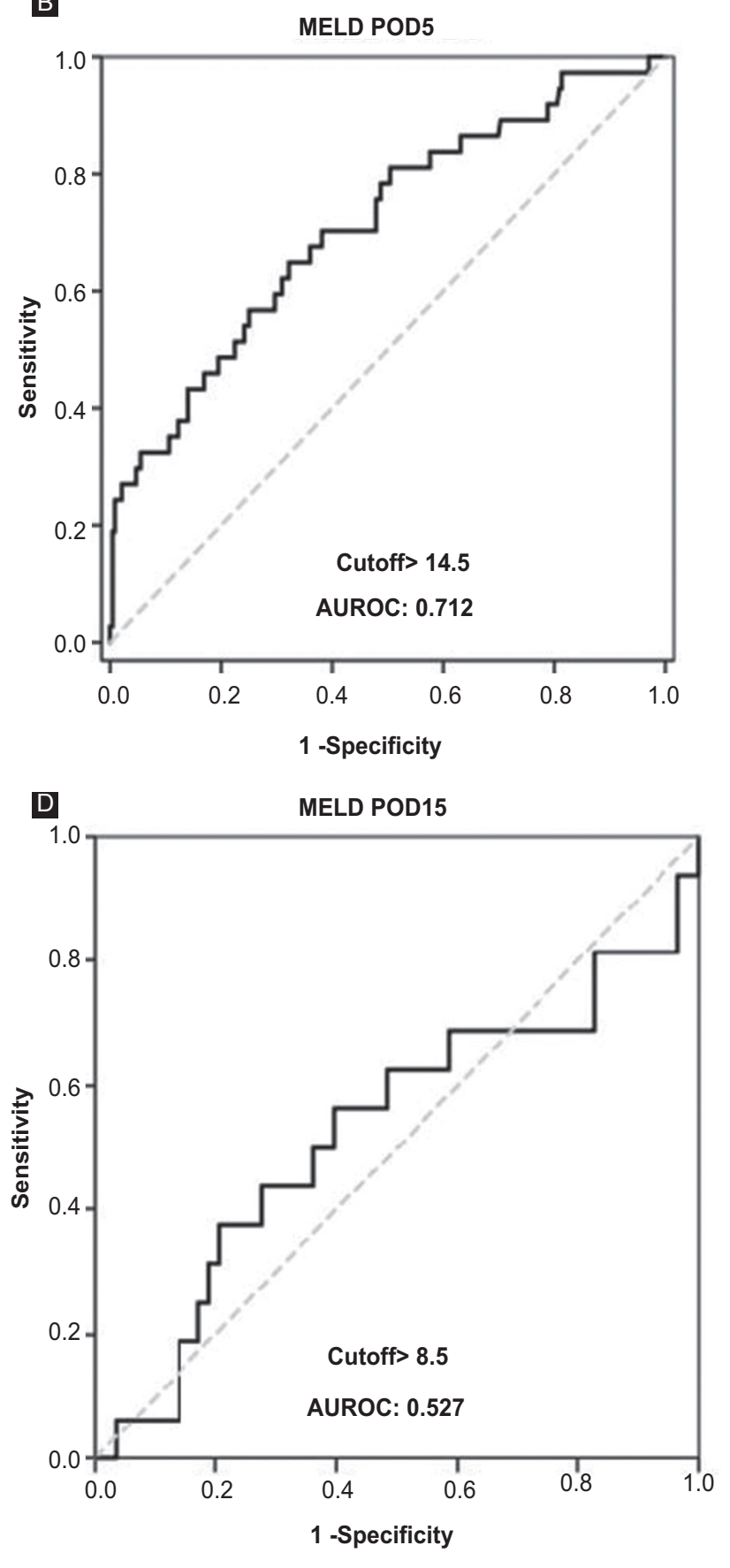

Figure 2 The AUROC for MELD score prior to transplantation (A) and on POD5 (B), POD9 (C) and POD15 (D) in relation to survival over 1 year of follow up AUROC, area under the receiver operating characteristic curve; MELD, model for end-stage liver disease; POD postoperative day

While postoperative MELD score can be affected by various factors independent of liver dysfunction, the parameters used in the MELD score can represent the overall well-being of the patient and therefore correlate with survival. In addition, our data showed that there was a significant correlation between mortality and patients' creatinine and INR. Therefore, the biggest effect was due to both liver and not only liver-related parameters. It should be noted that the findings presented in this study highlight the fact that conditions following Annals of Gastroenterology 32 calculation of post-LT MELD score may be responsible for later complications, including mortality. We measured MELD scores daily, which helped us identify the importance of MELD scores measured between the $5^{\text {th }}$ and $15^{\text {th }}$ postoperative days and we found that the MELD score in this population indicated a higher risk of mortality. These findings were important, as they assisted in the identification of possible underlying factors not related to the preoperative condition of either recipient or donor. Parameters such as bilirubin and INR directly reflect 
Table 3 The main causes of death among 90 patients who died after liver transplantation

\begin{tabular}{lc}
\hline Cause & $\mathrm{N}(\%)$ \\
\hline Sepsis & $41(41.8)$ \\
Cardiac event & $10(7.2)$ \\
\hline Intraoperative bleeding & $5(5.1)$ \\
Thrombosis & $6(4.3)$ \\
Renal failure & $5(3.6)$ \\
\hline ICH & $4(2.9)$ \\
\hline Graft dysfunction & $1(0.7)$ \\
\hline GVHD & $1(0.7)$ \\
\hline ARDS & $2(1.4)$ \\
PSC reoccurrence & $2(1.4)$ \\
\hline Pneumonia & $6(4.3)$ \\
Brain death & $1(0.7)$ \\
\hline DIC & $5(6.1)$ \\
\hline Gastrointestinal bleeding & $1(0.7)$ \\
\hline
\end{tabular}

ICH, intracerebral hemorrhage; GVHD, graft versus host disease; ARDS, acute respiratory distress syndrome; PSC, primary sclerosing cholangitis; DIC, disseminated intravascular coagulatio

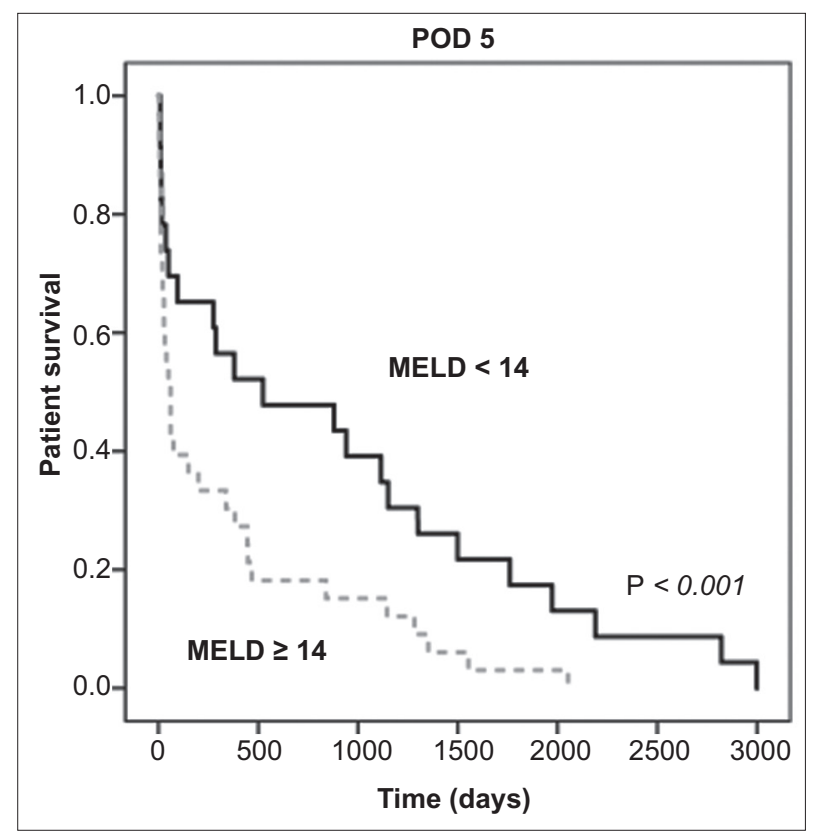

Figure 3 The Kaplan-Meier curve for patient survival after liver transplantation in the group with $M E L D \geq 14$ and the group with $\mathrm{MELD}<14$. The differences in survival between the two groups were compared using the log-rank test. $\mathrm{N}=826$ patients $M E L D$, model for end-stage liver disease

liver function, while creatinine is not a direct reflector of liver function, but can be an indicator of impaired kidney function secondary to hepatorenal syndrome. However, creatinine also often reflects acute renal failure, which has been shown to be associated with early allograft dysfunction as well as being a poor prognostic factor following LT $[20,21]$. Future studies are needed to identify other risk factors that lead to the early allograft dysfunction characterized by the post-LT MELD score.

The measurement of MELD score after surgery has been reported by Rahbari et al [14], who demonstrated that the MELD score on POD5 was independently associated with a 2-fold increase in mortality after hepatic resection (odds ratio 2.06, 95\%CI 1.41-3.02), and that an AUROC value of 0.862 had excellent prognostic ability in predicting mortality. Likewise, another study done by Toshima et al [22] showed that the MELD score on POD2 with an optimal cutoff point of 19 was able to predict mortality and graft loss. Recently, Khandoga et al [23] reported that MELD on POD7 could reliably predict 1-year survival following LT (AUROC c-statistics: 0.73). The findings presented in our study generally support these data, and the predictive power of MELD score on POD5 (AUC: 0.712) could differentiate between survivors and non-survivors within 1 year post LT. In addition, these discrepancies in MELD score cutoff values, in terms of discriminatory performance, may be due to heterogeneity across different study populations. For example, Toshima et al [22] reported that the mean MELD score prior to transplantation was 17 , whereas in our patient population we had a higher mean pre-LT MELD score of 21 . It should be noted that the change in MELD (delta MELD) may provide a better evaluation of patients' status than absolute MELD score.

Death occurs more commonly within the first year following LT. The major risk factors responsible for the cause of death include primary graft dysfunction, errors in surgical technique, biliary complications as well as infectious complications [24]. These well-established complications may occur before, during or postoperative periods. The use of Cox regression analyses in this study revealed several additional risk factors (such as operation duration, pre-LT MELD, and MELD on POD5) that were significantly associated with patient survival and should be taken into consideration regarding the precise assessment of patient status following LT. These findings further support this notion that measurement of post-LT MELD score can help clinicians to a prompt decision as to whether there is a need for early re-transplantation. Moreover, neither the cold nor the warm ischemia time played a key role in predicting mortality. This finding may be partly due to lower intergroup variations in our patient population.

The study has several limitations. First, its retrospective nature means that unobserved potential confounders may exist that were not controlled for in the analyses. For example we were not able to assess the role of post-LT intensive care unit stay, pre- and post-LT patient care, and rehabilitation in mortality outcomes. Moreover, although we used a relatively large database, the causal relationship between variables may not be generalizable to larger populations because of the single-center design of the current study. Therefore, findings presented in this study should interpreted in the context of its limitations.

In conclusion, immediate postoperative MELD scores after LT may hold value in predicting mortality and could be used as a tool for the postoperative risk assessment of patients. Consequently, in the management of patients with high postoperative MELD scores, warning signs of infection, intra-abdominal bleeding or primary graft dysfunction should trigger the initiation of appropriate therapeutic 
8 H. Dashti et al

Table 4 The association between variables and risk of mortality using logistic regression analysis

\begin{tabular}{|c|c|c|c|c|c|c|}
\hline \multirow[t]{2}{*}{ Variable } & \multicolumn{3}{|c|}{ Univariate logistic regression } & \multicolumn{3}{|c|}{ Multivariate logistic regression } \\
\hline & OR & $95 \% \mathrm{CI}$ & $\mathrm{P}^{*}$ & OR & $95 \% \mathrm{CI}$ & $\mathrm{P}$ \\
\hline Male recipient & 1.187 & $0.85-1.63$ & 0.309 & - & - & - \\
\hline Donors' age & 0.98 & $0.96-0.99$ & 0.007 & - & - & - \\
\hline Recipients' age & 0.99 & $0.98-1.001$ & 0.994 & - & - & - \\
\hline Pre-transplant Child-Pugh score & 0.93 & $0.85-1.01$ & 0.111 & - & - & - \\
\hline Pre-transplant MELD score & 0.93 & 0.91-0.95 & $<0.001$ & - & - & - \\
\hline POD5 MELD score & 0.91 & $0.87-0.95$ & $<0.001$ & 0.94 & $0.89-0.98$ & 0.014 \\
\hline POD9 MELD score & 0.92 & $0.88-0.97$ & 0.003 & - & - & - \\
\hline No. of PRC transfusions & 0.86 & $0.83-0.91$ & $<0.001$ & 0.98 & $0.91-1.05$ & 0.58 \\
\hline No. of platelet transfusions & 0.92 & $0.83-0.96$ & $<0.001$ & - & - & - \\
\hline No. of FFP transfusions & 0.91 & $0.88-0.95$ & $<0.001$ & - & - & - \\
\hline Cold ischemic time (min): $\geq 300$ & 0.99 & $0.98-1.02$ & 0.001 & 0.99 & $0.96-1.004$ & 0.71 \\
\hline Warm ischemic time $(\mathrm{min}): \geq 35$ & 0.99 & $0.98-1.008$ & 0.604 & - & - & - \\
\hline Operation duration: $\geq 350$ & 0.99 & $0.95-1.11$ & $<0.001$ & 0.99 & $0.99-1.003$ & 0.038 \\
\hline Post-operation ICU stay (days): $\geq 5$ & 1.012 & $0.99-1.02$ & 0.199 & - & - & - \\
\hline
\end{tabular}

${ }^{{ }^{2}} \mathrm{P}<0.05$ is statistically significant

OR, odds ratio; CI, confidence interval; POD, postoperative day; MELD, model for end-stage liver disease; PRC, packed red cells; FFP, fresh frozen plasma;

ICU, intensive care unit

Table 5 Cox regression analysis of risk factors influencing overall survival

\begin{tabular}{|c|c|c|c|c|c|c|}
\hline \multirow[t]{2}{*}{ Variables } & \multicolumn{3}{|c|}{ Univariate analysis } & \multicolumn{3}{|c|}{ Multivariate analysis } \\
\hline & HR & $95 \% \mathrm{CI}$ & $\mathrm{P}$-value & $\mathrm{HR}$ & $95 \% \mathrm{CI}$ & P-value \\
\hline Recipient's gender, male & 1.24 & $0.86-1.76$ & 0.336 & - & - & - \\
\hline Donor's gender, male & 0.91 & $0.66-1.25$ & 0.79 & - & - & - \\
\hline Cirrhosis etiology & & & & - & - & - \\
\hline Hepatitis B & 0.17 & $0.04-0.66$ & 0.011 & - & - & - \\
\hline Hepatitis C & 0.27 & $0.07-0.98$ & 0.048 & - & - & - \\
\hline Autoimmune & 0.31 & $0.08-1.02$ & 0.36 & - & - & - \\
\hline NASH & 1.28 & $0.25-5.34$ & 0.76 & - & - & - \\
\hline Pre-transplant Child score & 1.35 & $0.92-1.99$ & 0.117 & & & \\
\hline Pre-transplant MELD score & 1.034 & $1.005-1.065$ & 0.023 & 1.064 & $1.025-1.104$ & 0.001 \\
\hline POD5 MELD score: $\geq 14$ & 1.071 & $1.036-1.107$ & $<0.001$ & 1.83 & $1.07-3.12$ & 0.026 \\
\hline POD9 MELD score: $\geq 11.5$ & 1.062 & $1.024-1.102$ & 0.001 & - & - & - \\
\hline No. of PRC transfusion: $\geq 5$ & 0.99 & $0.95-1.03$ & 0.75 & - & - & - \\
\hline Biliary complications & 0.89 & $0.82-0.97$ & 0.021 & 0.78 & $0.45-1.07$ & 0.32 \\
\hline $\mathrm{CBD}$ anastomosis: end-to-end/Roux-en-Y & 0.76 & $0.41-1.42$ & 0.537 & - & - & - \\
\hline Cold ischemic time (min): $\geq 300$ & 0.99 & $0.91-1.01$ & 0.106 & - & - & - \\
\hline Warm ischemic time (min): $\geq 35$ & 0.98 & $0.97-0.99$ & $<0.001$ & - & - & - \\
\hline Operation duration: $\geq 350$ & 0.99 & $0.93-1.12$ & 0.034 & 1.004 & $1.003-1.006$ & 0.013 \\
\hline Post-operation ICU stay (days): $\geq 5$ & 0.99 & $0.98-1.004$ & 0.611 & - & - & - \\
\hline
\end{tabular}

HR, hazard ratio; CI, confidence interval; NASH, nonalcoholic steatohepatitis; CBD, common bile duct; POD, postoperative day; MELD, model for end-stage liver disease; PRC, packed red cells; ICU, intensive care unit 
interventions and careful monitoring. Moreover, the utility of early postoperative MELD scores as a prognostic model may enable physicians to predict the need for re-transplantation during patients' clinical course. Future multicenter cohort studies are warranted to confirm these findings.

\section{References}

1. Murray KF, Carithers RL Jr; AASLD. AASLD practice guidelines: evaluation of the patient for liver transplantation. Hepatology 2005;41:1407-1432.

2. Gad EH, Alsebaey A, Lotfy M, Eltabbakh M, Sherif AA. Complications and mortality after adult to adult living donor liver transplantation: a retrospective cohort study. Ann Med Surg (Lond) 2015;4:162-171.

3. Derbisz K, Nylec M, Chrząszcz P, et al. Recipient-related preoperative and intraoperative risk factors for primary graft dysfunction after orthotopic liver transplantation. Transplant Proc 2018;50:2018-2021.

4. Manzia TM, Angelico R, Toti L, et al. Longterm survival and cost-effectiveness of immunosuppression withdrawal after liver transplantation. Liver Transpl 2018;24:1199-1208.

5. Wan P, Yu X, Xia Q. Operative outcomes of adult living donor liver transplantation and deceased donor liver transplantation: a

\section{Summary Box}

\section{What is already known:}

- The model for end-stage liver disease (MELD) score is considered as a reliable prognostic model for mortality prediction among patients on the liver transplantation (LT) waiting list

- The occurrence of postoperative complications depends on several donor and recipient-related risk factors that contribute to a substantial impact on outcomes and costs

- Postoperative patient survival is affected by preoperative factors related to both donor and recipient, including recipient age and quality of the donor liver graft, as well as surgical procedure complexity

\section{What the new findings are:}

- The immediate postoperative MELD scores after LT could predict mortality

- MELD score on postoperative day (POD) 5 was independently associated with mortality

- The optimal cutoff value of 14.5 was obtained for MELD score on POD5, which significantly differentiated between surviving and nonsurviving patients with a sensitivity of $69.8 \%$ and a specificity of $57.2 \%$ systematic review and meta-analysis. Liver Transpl2014;20:425-436

6. Gil E, Kim JM, Jeon K, et al. Recipient age and mortality after liver transplantation: a population-based cohort study. Transplantation 2018;102:2025-2032.

7. Braun HJ, Ascher NL. The impact of renal insufficiency after liver transplantation. Transplantation 2018;102:1033-1034.

8. Lim HS, Kim HC, Park YH, Kim SK. Evaluation of malnutrition risk after liver transplantation using the nutritional screening tools. Clin Nutr Res 2015;4:242-249.

9. Bastos-Neves D, Salvalaggio PRO, Almeida MD. Risk factors, surgical complications and graft survival in liver transplant recipients with early allograft dysfunction. Hepatobiliary Pancreat Dis Int 2019 Feb 25 [Epub ahead of print]. doi: 10.1016/j.hbpd.2019.02.005.

10. Malinchoc M, Kamath PS, Gordon FD, Peine CJ, Rank J, ter Borg PC. A model to predict poor survival in patients undergoing transjugular intrahepatic portosystemic shunts. Hepatology 2000;31:864-871.

11. Wiesner R, Edwards E, Freeman R, et al; United Network for Organ Sharing Liver Disease Severity Score Committee. Model for end-stage liver disease (MELD) and allocation of donor livers. Gastroenterology 2003;124:91-96.

12. Trieu JA, Bilal M, Hmoud B. Factors associated with waiting time on the liver transplant list: an analysis of the United Network for Organ Sharing (UNOS) database. Ann Gastroenterol 2018;31:84-89.

13. Chen RC, Wang XD, Dong JZ, et al. A MELD-based nomogram for predicting 3-month mortality of patients with acute-on-chronic hepatitis B liver failure. Clin Chim Acta 2017;468:195-200.

14. Rahbari NN, Reissfelder C, Koch M, et al. The predictive value of postoperative clinical risk scores for outcome after hepatic resection: a validation analysis in 807 patients. Ann Surg Oncol 2011;18:3640-3649.

15. General Assembly of the World Medical Association. World Medical Association Declaration of Helsinki: ethical principles for medical research involving human subjects. J Am Coll Dent 2014;81:14-18.

16. Wiesner RH, McDiarmid SV, Kamath PS, et al. MELD and PELD: application of survival models to liver allocation. Liver Transpl 2001;7:567-580.

17. Cholongitas E, Marelli L, Shusang V, et al. A systematic review of the performance of the model for end-stage liver disease (MELD) in the setting of liver transplantation. Liver Transpl 2006;12:1049-1061.

18. Merion RM, Wolfe RA, Dykstra DM, Leichtman AB, Gillespie B, Held PJ. Longitudinal assessment of mortality risk among candidates for liver transplantation. Liver Transpl 2003;9:12-18.

19. Rostved AA, Lundgren JD, Hillingsø J, Peters L, Mocroft A, Rasmussen A. MELD score measured day 10 after orthotopic liver transplantation predicts death and re-transplantation within the first year. Scand J Gastroenterol 2016;51:1360-1366.

20. Zand MS, Orloff MS, Abt P, et al. High mortality in orthotopic liver transplant recipients who require hemodialysis. Clin Transplant 2011;25:213-221.

21. Cabezuelo JB, Ramírez P, Ríos A, et al. Risk factors of acute renal failure after liver transplantation. Kidney Int 2006;69:1073-1080.

22. Toshima T, Ikegami T, Kimura K, et al. Application of postoperative model for end-stage liver disease scoring system for evaluating liver graft function after living donor liver transplantation. Transplant Proc 2014;46:81-86.

23. Khandoga A, Iskandarov E, Angele M, et al. Model for end-stage liver disease score in the first 3 weeks after liver transplantation as a predictor for long-term outcome. Eur J Gastroenterol Hepatol 2016;28:153-158.

24. Watt KD, Pedersen RA, Kremers WK, Heimbach JK, Charlton MR. Evolution of causes and risk factors for mortality post-liver transplant: results of the NIDDK long-term follow-up study. Am J Transplant 2010;10:1420-1427. 